\title{
Current trends in the management of proximal humerus fractures in elderly patients
}

\author{
Claudio Ascani, Marco Scacchi, Daniele Passaretti, \\ Gianluca Bullitta, Mauro De Cupis, Piergiorgio Calella, \\ Lucagiuseppe Bruno, Massimiliano Trombaccia
}

Trauma Orthopedic Center A. Alesini, Rome, Italy
Received: April 2, 2021

Accepted: April 6, 2021

Correspondence

Claudio Ascani

Trauma Orthopedic Center A. Alesini, via San Nemesio 21, 00145 Rome, Italy

E-mail: claudio.ascani@ aslroma2.it

Conflict of interest

The authors have no conflict of interest to declare.

How to cite this article: Ascani C, Scacchi M, Passaretti D, et al. Current trends in the management of proximal humerus fractures in elderly patients. Lo Scalpello Journal 2021;35:1-5. https://doi.org/10.36149/0390-5276-210

(C) Ortopedici Traumatologi Ospedalieri d'Italia (O.T.O.D.I.) 2021

\section{(c) (1) (8) $\Theta$}

This is an open access article distributed in accordance with the CC-BY-NC-ND (Creative Commons Attribution-NonCommercial-NoDerivatives 4.0 International) license. The article can be used by giving appropriate credit and mentioning the license, but only for non-commercial purposes and only in the original version. For further information: https://creativecommons.org/licenses/by-nc-nd/4.0/deed.en

\begin{abstract}
SUMMARY
Proximal humeral fractures are among the most common fractures in the elderly. Along with the increasing life expectancy of the Western population, the incidence of these fractures is rising rapidly, with osteoporosis as an important factor, affecting mostly active persons aged 60 and older. In such patients, proximal humeral fracture can potentially affect independence and deteriorate the quality of life.

Currently, several options are available for the treatment of proximal humerus fractures in elderly patients. The main treatments include open reduction and internal fixation, percutaneous closed fixation, and intramedullary nailing or total shoulder arthroplasty, depending on multiple variables including patient age, comorbidities, activity level, functional demands, and goals/expectations of treatment.
\end{abstract}

Key words: proximal humerus fractures, elderly, osteoporosis

\section{Introduction}

Proximal humeral fractures are among the most common fractures in the elderly ${ }^{1}$. Along with the increasing life expectancy of the Western population, the incidence of these fractures is rising rapidly, with osteoporosis as an important factor ${ }^{2,3}$, affecting mostly active persons aged 60 and older ${ }^{1}$. Around $90 \%$ of these patients live independently at home and therefore, a proximal humeral fracture can potentially affect independence and deteriorate the quality of life in the elderly.

Approximately $80 \%$ of proximal humerus fractures have a stable configuration and may be treated conservatively with satisfactory results, especially in low demanding elderly patients. The remaining cases may be treated surgically. However, whether reduction and fixation or primary shoulder arthroplasty may be best indicated in these cases is still matter of debate ${ }^{4}$.

Conservative treatment may represent an option even for displaced or comminuted proximal humerus fractures in patients older than 85 years of age affected by severe osteoporosis, cognitive impairment, or significant comorbidities ${ }^{5,6}$.

In any case, the proper indication for conservative or surgical treatment should take into account several factors: expected outcome, patient's compliance, surgeon's training, and experience. However, consensus is thus still lacking on the most appropriate treatment for this type of fracture in elderly patients and most studies 
do not take into account functioning in daily life and social participation, rather focusing mainly on range of motion and functional and radiological outcomes ${ }^{1,7,8}$.

Osteoporosis may significantly influence the fixation of proximal humerus fracture, especially in a comminuted fracture pattern. Therefore, the choice of the correct surgical technique and implant is crucial when facing these kinds of fractures.

Currently, several techniques are available for the treatment of proximal humerus fractures in elderly patients. The same fracture pattern can be treated nonoperatively, with open or closed fixation, or with arthroplasty, depending on multiple variables including patient age, comorbidities, activity level, functional demands, and goals/expectations of treatment.

\section{Locking plate fixation}

Plate fixation represents one of the most common treatments for proximal humerus fractures. In particular, the introduction of locked plating has allowed for the creation of stable constructs even in osteoporotic bone with satisfactory results ${ }^{9}$. A meta-analysis of locking plate fixation for proximal humerus fractures showed an average overall constant score of 74 with minimum 18-month follow-up, 79 for 2-part fractures, 72 for 3-part fractures, and 66 for 4 -part fractures ${ }^{10}$. Despite improvements in plate technology, complications and reoperation rates remain a concern. Zhang et al., in a study on 27,017 patients surgically treated for proximal humerus fractures, reported a higher readmission rate for ORIF (29\%) compared to other techniques such as reverse shoulder arthroplasty (20\%) and hemiarthroplasty (16\%) ${ }^{11}$.

The authors showed that the causes of complications are multifactorial, and involve patient-related factors (e.g., comorbid conditions, smoking), fracture-specific factors (e.g., bone quality, comminution, fracture pattern), surgical factors (e.g., plate/ screw placement, reduction quality), and postoperative rehabilitation. Among the main and common complications, there were varus malunion (16.3\%) followed by avascular necrosis $(10.8 \%)$ (Fig. 1), screw cutout $(7.5 \%)$, subacromial impingement $(4.8 \%)$, and infection $(3.5 \%)$. Screw cutout was the most common reason for revision surgery ${ }^{10}$.

The screw position represents an important factor for the stability of the fracture. In particular, screw position is most important in the calcar. Placement of calcar screws is critical in metaphyseal comminution to achieve medial column support ${ }^{7}$. The placement of the calcar screws in a more distal position, as opposed to a more proximal position within the head, is critical for improved biomechanical stability ${ }^{12}$.

In a biomechanical study using 11 matched pairs of human cadaveric proximal humerus fractures, medial calcar comminution decreased the mean load to failure by $48 \%$ and energy to failure by $44 \%$. The use of calcar screws increased the mean load to failure by $31 \%$ and energy to failure by $44 \%$, underscoring the importance of calcar screw support in cases of medial comminution ${ }^{13}$.
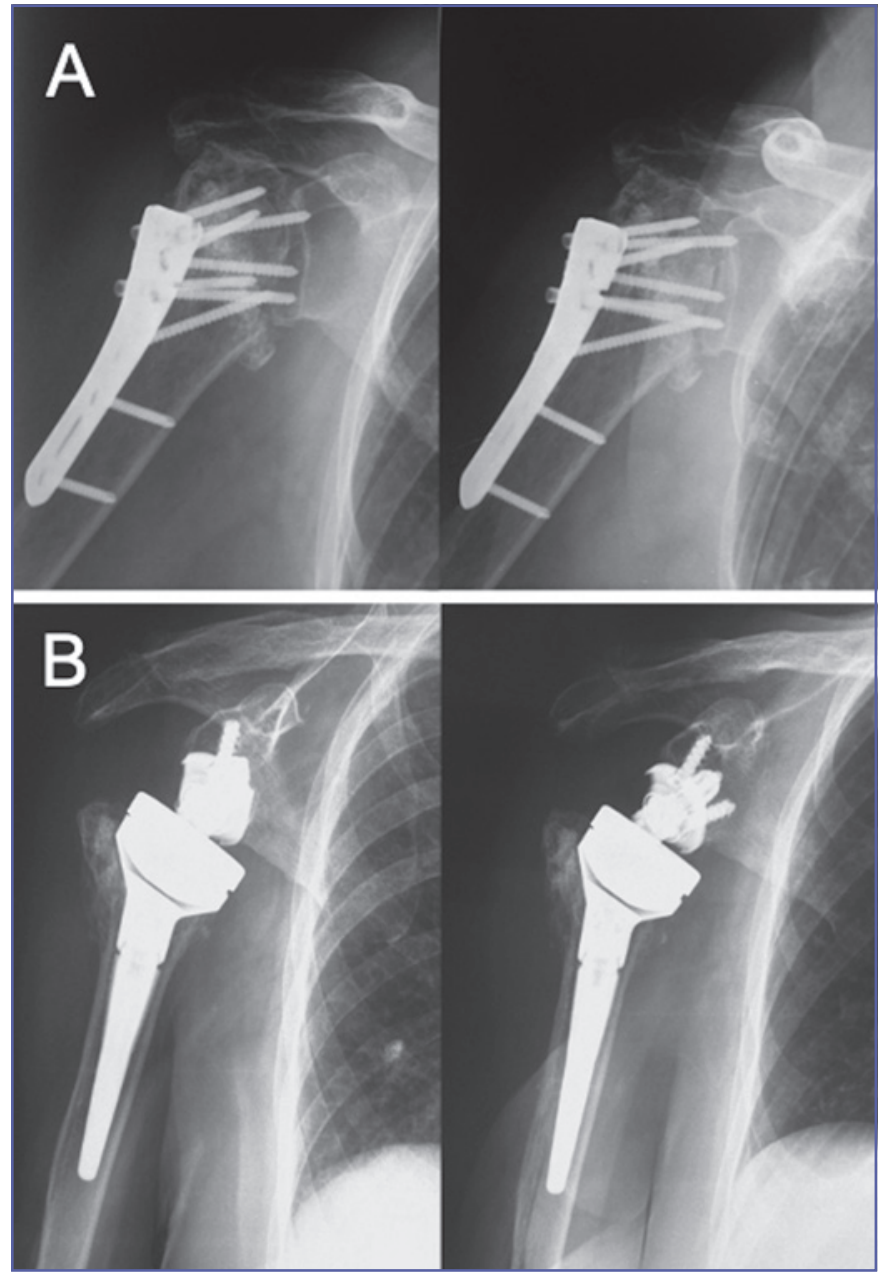

Figure 1. A) avascular necrosis following open reduction and internal fixation of a proximal humerus fracture; B) revision with reverse total shoulder arthroplasty.

In order to improve the biomechanical stability of locked proximal humerus plates, cement augmented screws have been introduced for osteoporotic proximal humerus fractures. Varga et al, in a computer simulation, showed that cement augmentation of the screw tips in plate fixation of unstable three-part fractures can alleviate the peri-implant strain, which suggests a reduced risk of cut-out failure. The number as well as the specific configuration of the augmented screws was highly influential to the achieved benefit and optimal screw selection may be patient-specific. However, augmenting the calcar screws consistently provided the largest improvement and augmentation of the screws with posterior tips was also beneficial. Further investigation through additional simulations, in vitro testing, and prospective clinical trials involving augmentation of specific screws is required to better define optimal techniques and evaluate patient benefit ${ }^{14}$. The use of intramedullary bone graft may also be an alternative, especially in cases of proximal humerus fractures with severe 
metadiaphyseal comminution by creating a medial column of support and preventing varus collapse of the humeral head. The use of a fibular strut allograft and iliac crest autograft bone have been described. Biomechanical studies showed superior biomechanical stability when using the fibular strut compared with plate alone ${ }^{7}$. However, it may be argued that the cost-benefit ratio in elderly patients could be disadvantageous.

\section{Closed reduction and percutaneous fixation}

Closed reduction and percutaneous pinning (CRPP) may be considered as an alternative to open reduction and internal fixation in specific fracture patterns and in selected patients. Pin configuration is a significant factor in order to enhance mechanical stability, which is improved by biplanar fixation and by increasing the number of pins engaging the cortex ${ }^{15}$.

Indications for CRPP include 2-part fractures of the surgical neck, isolated greater tuberosity fractures, 3-part fractures of the surgical neck with involvement of the greater tuberosity, and 4-part valgus impacted fractures ${ }^{8}$. The advantages of these techniques are possible preservation of the vascular supply to bone fragments, shorter duration of intervention, maintenance of fracture hematoma, scarce blood loss, and the possibility of surgery with brachial plexus block ${ }^{15}$. In a study on 113 patients with 2-part PHFs, Tamimi et al. reported better outcomes with CRPP in patients of all ages and better functional results compared to intramedullary nailing in elderly patients ${ }^{16}$.

On the other hand, Gupta et al. published a review on 4500 patients, reportinga considerably higher complication rates with CRPP compared to ORIF, hemiarthroplasty, and reverse shoulder arthroplasty. Complications observed with CRPP included humeral head necrosis $(11.7 \%)$, pin migration/breakage $(4.1 \%)$, superficial infection $(4.1 \%)$, malunion $(3 \%)$, neurologic injuries $(1.5 \%)$, and nonunion and deep infection $(1 \%)^{17}$.

The poor quality of the bone in osteoporotic elderly fractures may predispose to pin migration, loosening, and loss of reduction. However, recent technical advances enhanced the results obtained with this technique. Blonna et al. suggested the use of full threated pins augmented by an external frame. The authors prospectively studied 42 patients treated with conventional pinning ( $2.5 \mathrm{~mm}$ terminally threaded pins) and 49 patients treated with the hybrid technique $(2.5 \mathrm{~mm}$ pins characterized by a $7 \mathrm{~cm}$ thread augmented with external fixator). They observed a significant reduction in complications and revision rates in the hybrid group ${ }^{18}$. Gumina et al. compared two different fixation constructs with threaded locked wires showing that the functional and radiologic results obtained after treatment of 3-part proximal humeral fractures with percutaneous fixation or locking plates were similar, but the percentage of major complications after percutaneous treatment were lower and the maintenance of reduction and fracture stability in CRPP is influenced by the biomechanical construct and number of pins engaging the cortex ${ }^{15}$.

\section{Intramedullary nail fixation}

Intramedullary nailing may also represent a valid alternative for treatment of proximal humerus fractures in the elderly patient. In particular, last generation nails with multidirectional locked cancellous proximal screws allows more stable constructs. Moreover, straight nails with a medial entry point on the humeral head helps to preserve the rotator cuff footprint allowing better functional results and lower complication rates ${ }^{1,8}$.

Recent studies assert that results achieved with latest generation proximal humerus nails are comparable to those reported with locking plates ${ }^{19,20}$. Furthermore, in a comparative randomized study of 2-part proximal humerus fractures treated with proximal intramedullary nail or plates, Zhu et al. reported complication rates of 4 and $31 \%$, respectively ${ }^{21}$.

When performing intramedullary nailing fixation of these fractures, it is preferable to use straight nails. The entry point should be at the center of the humeral head in consideration of the better quality of the subchondral bone, which enhance the stability of the nail. Moreover, the medial entry point allows to split the muscular portion of the supraspinatus tendon, avoiding disturbances to the tendon.

In our experience, the nail is a proper implant for treatment of different types of proximal humeral fractures (Fig. 2). It has some advantages because of its minimally invasive technique: less soft-tissue damage and shorter operation time. Additionally, several tests have shown the biomechanical superiority of the intramedullary nail. This can be advantageous for elderly osteoporotic patients. Functional results seem to be determined mainly by fracture type and patient age. Based on the literature, the use of latest generation proximal humeral nails to treat proximal humeral fractures can be recommended.

\section{Reverse total shoulder arthroplasty}

Hemiarthroplasty has been the historical treatment for unreconstructible fractures of the proximal humerus ${ }^{22}$. This has changed over the recent years in favor of reverse total shoulder arthroplasty (RTSA) for treatment of unreconstructible proximal humerus fractures in the elderly (Fig. 3). The rate of RTSA for treatment of proximal humerus fractures has almost doubled from 2011 to 2013 , rising from $13 \%$ of operative cases to $24 \%$ of operative cases, with hemiarthroplasty dropping from 28 to $21 \%{ }^{22}$. The main reason for this phenomenon is that the outcome seems to be much more predictable, compared to hemiarthroplasty, because it does not depend on ingrowth of tuberosities to such an extent ${ }^{7}$.

Although greater tuberosity healing is not critical for a successful result, healing of the greater tuberosity in RTSA is important for improved external rotation ${ }^{23}$.

In a recent study, Fraser et al. compared the results of open reduction and internal fixation with plate and reverse shoulder arthroplasty. At 2-year follow-up, the data suggested an advan- 


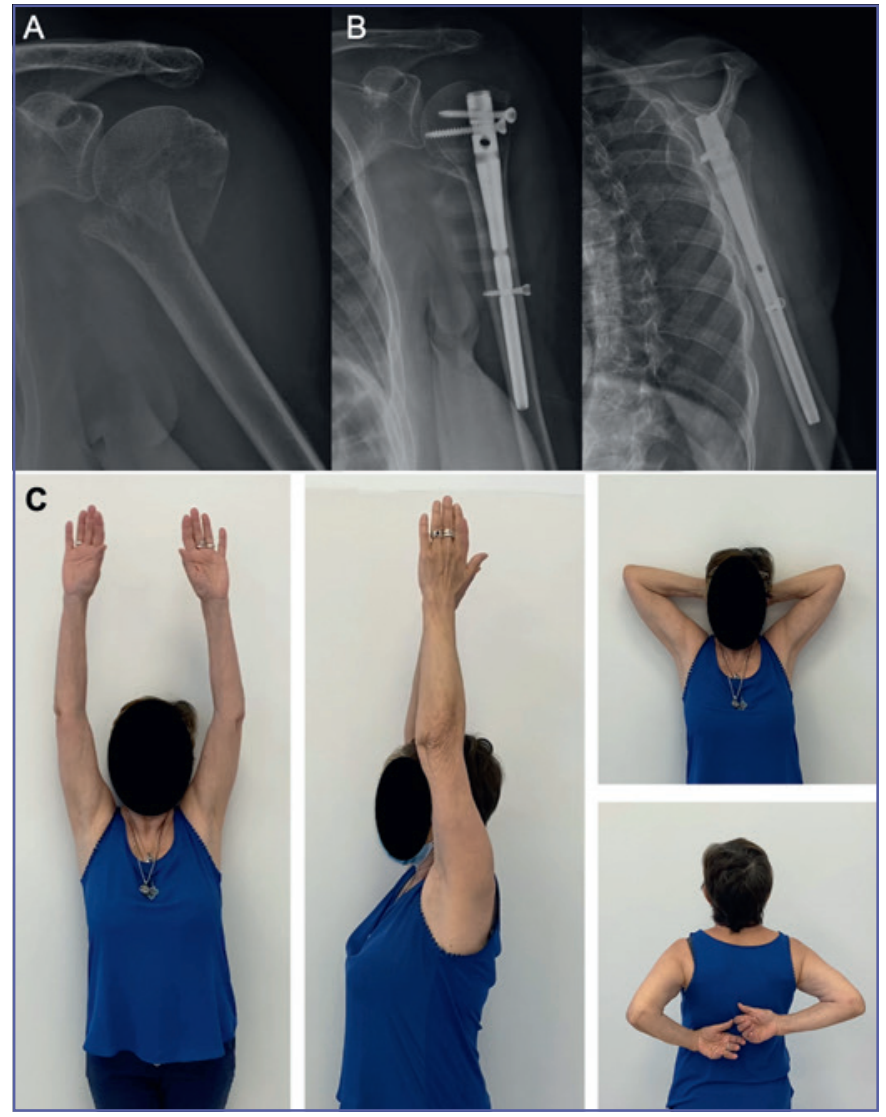

Figure 2. A) 3-part proximal humerus fracture in a 67-year-old woman treated with intramedullary nailing; $\mathrm{B})$ radiological and $\mathrm{C}$ ) clinical outcomes at 12 months.

tage of RTSA over proximal plating in the treatment of displaced OTA/AO type-B2 and C2 proximal humeral fractures in elderly patients ${ }^{24}$.

Lopiz et al. compared the results of RTSA to non-operative treatment for 3- or 4-part proximal humeral fractures in elderly patients. Their study suggested that at short-term follow-up, there are no clinical benefits of RTSA over non-operative treatment except pain perception. However, the study had some limitations. The short follow-up may have influenced the results. In fact, a longer follow-up period could detect possible differences in the complication rates of RTSA and in the development of future complications including osteonecrosis and post-traumatic arthritis. Moreover, the results may have been impaired by comorbidities of the patients analyzed which may influence the final outcome and perceived quality of life among therapeutic options ${ }^{25}$.

\section{Conclusions}

Treatment of proximal humerus fracture in patients with osteo-
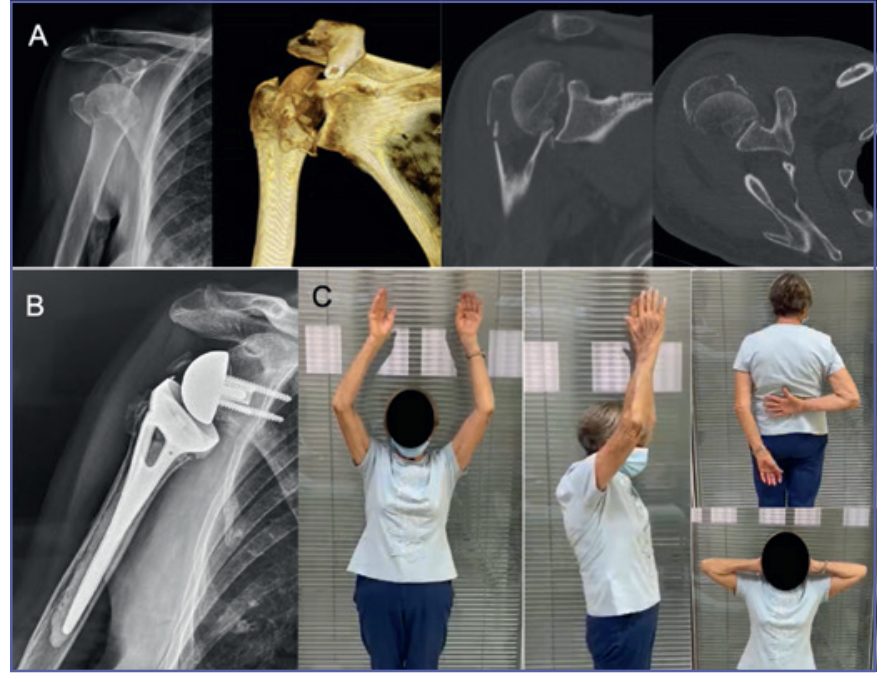

Figure 3. A) preoperative $X$-ray and CT scans of a 4-part proximal humerus fracture in a 75-year-old woman; B) radiological and $\mathrm{C}$ ) clinical outcomes at 12 months.

porotic bone remains a challenge in terms of when and how to operate. Several treatments are available, but therapy should be ideally individualized on the basis of the patient's functional demands and biologic prognostic factors related to the characteristics of the fracture.

\section{References}

Wendt KW, Jaeger M, Verbruggen J, et al. ESTES recommendations on proximal humerus fractures in the elderly. Eur J Trauma Emerg Surg 2020. https://doi.org/10.1007/s00068-020-01437-7 [Epub ahead of print]

2 Palvanen M, Kannus P, Niemi S, et al. Update in the epidemiology of proximal humeral fractures. Clin Orthop Relat Res 2006;442:87-92.

Johnell O, Kanis J. Epidemiology of osteoporotic fractures. Osteoporos Int 2005;16(Suppl 2):6-10.

4 Murray IR, Amin AK, White TO, et al. Proximal humeral fractures: current concepts in classification, treatment and outcomes. J Bone Jt Surg - Ser B 2011;93B:1-11.

5 Schumaier A, Grawe B. Proximal humerus fractures: evaluation and management in the elderly patient. Geriatr Orthop Surg Rehabil 2018;9:215145851775051.

6 Fjalestad T, Hole MØ. Displaced proximal humeral fractures: operative versus non-operative treatment - a 2-year extension of a randomized controlled trial. Eur J Orthop Surg Traumatol 2014;24:1067-1073.

7 Stone MA, Namdari S. Surgical considerations in the treatment of osteoporotic proximal humerus fractures. Orthop Clin North Am 2019;50:223-231. https://doi.org/10.1016/j.ocl.2018.10.005

8 Murena L, Canton G, Ratti C, et al. Indications and results of osteosynthesis for proximal humerus fragility fractures in elderly patients. Orthop Rev (Pavia) 2020;12. 
9 Seide K, Triebe J, Faschingbauer M, et al. Locked vs unlocked plate osteosynthesis of the proximal humerus - a biomechanical study. Clin Biomech 2007;22:176-182.

10 Sproul RC, Iyengar JJ, Devcic Z, et al. A systematic review of locking plate fixation of proximal humerus fractures. Injury 2011;42:408-413. http://dx.doi.org/10.1016/j.injury.2010.11.058

11 Zhang AL, Schairer WW, Feeley BT. Hospital readmissions after surgical treatment of proximal humerus fractures: is arthroplasty safer than open reduction internal fixation? Clin Orthop Relat Res 2014;472:2317-2324.

12 Mehta S, Chin M, Sanville J, et al. Calcar screw position in proximal humerus fracture fixation: don't miss high! Injury 2018;49:624-629. http://dx.doi.org/10.1016/j.injury.2018.02.007

13 Ponce BA, Thompson KJ, Raghava P, et al. The role of medial comminution and calcar restoration in varus collapse of proximal humeral fractures treated with locking plates. J Bone Jt Surg - Ser A 2013;95:1-7.

14 Varga P, Inzana JA, Fletcher JWA, et al. Cement augmentation of calcar screws may provide the greatest reduction in predicted screw cut-out risk for proximal humerus plating based on validated parametric computational modelling. Bone Jt Res 2020;9:534-542.

15 Gumina S, Candela V, Giannicola G, et al. Complex humeral head fractures treated with blocked threaded wires: maintenance of the reduction and clinical results with two different fixation constructs. J Shoulder Elb Surg 2019;28:36-41. https://doi. org/10.1016/j.jse.2018.06.034

16 Tamimi I, Montesa G, Collado F, et al. Displaced proximal humeral fractures: when is surgery necessary? Injury 2015;46:1921-1929.

17 Gupta AK, Harris JD, Erickson BJ, et al. Surgical management of complex proximal humerus fractures - a systematic review of 92 studies including 4500 patients. J Orthop Trauma 2015;29:54-59.
18 Blonna D, Castoldi F, Scelsi M, et al. The hybrid technique: potential reduction in complications related to pins mobilization in the treatment of proximal humeral fractures. J Shoulder Elb Surg 2010;19:1218-1229. http://dx.doi.org/10.1016/j.jse.2010.01.025

19 Konrad G, Audigé L, Lambert S, et al. Similar outcomes for nail versus plate fixation of three-part proximal humeral fractures. Clin Orthop Relat Res 2012;470:602-609.

20 Lekic N, Montero NM, Takemoto RC, et al. Treatment of twopart proximal humerus fractures: intramedullary nail compared to locked plating. HSS J 2012;8:86-91.

${ }^{21}$ Zhu Y, Lu Y, Shen J, et al. Locking intramedullary nails and locking plates in the treatment of two-part proximal humeral surgical neck fractures: a prospective randomized trial with a minimum of three years of follow-up. J Bone Jt Surg - Ser A 2011;93:159-168.

22 Critchley O, McLean A, Page R, et al. Reverse total shoulder arthroplasty compared to stemmed hemiarthroplasty for proximal humeral fractures: a registry analysis of 5946 patients. J Shoulder Elb Surg 2020;29:2538-2547. https://doi.org/10.1016/j. jse.2020.04.005

23 Chun YM, Kim DS, Lee DH, et al. Reverse shoulder arthroplasty for four-part proximal humerus fracture in elderly patients: can a healed tuberosity improve the functional outcomes? J Shoulder Elb Surg 2017;26:1216-1221. http://dx.doi.org/10.1016/j. jse.2016.11.034

24 Fraser AN, Bjørdal J, Wagle TM, et al. Reverse shoulder arthroplasty is superior to plate fixation at 2 years for displaced proximal humeral fractures in the elderly: a multicenter randomized controlled trial. J Bone Jt Surg Am 2020;102:477-485.

25 Lopiz Y, Alcobía-Díaz B, Galán-Olleros M, et al. Reverse shoulder arthroplasty versus nonoperative treatment for 3- or 4-part proximal humeral fractures in elderly patients: a prospective randomized controlled trial. J Shoulder Elb Surg 2019;28:2259-2271. 